\title{
Internet of Things as a Logical Way to Tackle the Emerging COVID-19 Virus
}

\author{
G. Pius Agbulu ${ }^{1}$, H. Ndwabe ${ }^{2}$ \\ ${ }^{1}$ Acces Research Lab, Electronics and Instrumentation Engineering (EIE) \\ SRM Institute of Science and Technology, Chennai-603 203, \\ Tamil Nadu, India, godspowerpiusagbulu@gmail.com \\ ${ }^{2}$ Pharmaceutics Lab, SRM Institute of Science and Technology, Chennai-603 203, \\ Tamil Nadu, India, hamundwabe@gmail.com
}

\begin{abstract}
The outburst of the novel coronavirus (COVID-19) that has led to millions of deaths globally appears to be overwhelming. This virus has become a focus of worldwide consideration. The Internet of Things (IoT) can be well-thought-out as a practical way to forecast, monitor, and avert the emerging COVID-19 virus. Internet of Things represents a connected-network of smart objects, sensors, devices, and people over which information can be acquired and communicated via the cloud for trend and patterns analysis. This technology has gained immense research plethora in health care and industrial applications. The IoT revolution is reforming contemporary healthcare schemes through integrating high-tech, commercial, and societal views. This paper briefly examines the potential of IoT technology as a logical way to tackle COVID-19 by evolving healthcare systems from conventional to more personalized healthcare systems through which illness can be detected, treated, and supervised effortlessly.
\end{abstract}

Key words: COVID-19, Death, Internet of Things, Sensors, Virus.

\section{INTRODUCTION}

The Internet of Things (IoT) is revolutionizing present-day healthcare schemes by integrating high-tech, commercial, and societal views [1]. IoT has become a significant technology in modern-day healthcare applications as it presents cost-effectiveness, and cutting-edge user practices [2]. IoT is envisioned as a resolution to the contemporary worldwide challenge of COVID-19[3]. The symptoms of COVID-19 illness include cough, fatigue, and fever, which are crucial to identify promptly for treatments.

Typically, the virus gestation period spans between 1- 14 days, while the recovery-time spans from 6-41 days. The virus can transfer from a negative person with no visible symptoms.
Consequently, IoT technology's incorporation can be viewed as a safe and reliable direction toward handling the COVID-19 virus. Given the current worldwide condition, IoT -based surveillance technologies can be a major step towards controlling the COVID-19 pandemic [5-7]. Given the currently available infrastructures, the IoTmulti-functionality can help deal with the wave of the virus. Figure 1 illustrates the internet of things in present-day healthcare application. Primarily, IoT-enabled illness monitoring mechanisms can offer instantaneous data-availability and aid distant medical assistance [8-9]. The joint role of IoT and associated emerging technologies can aid prompt identification of the virus and implementation of preventive measures.

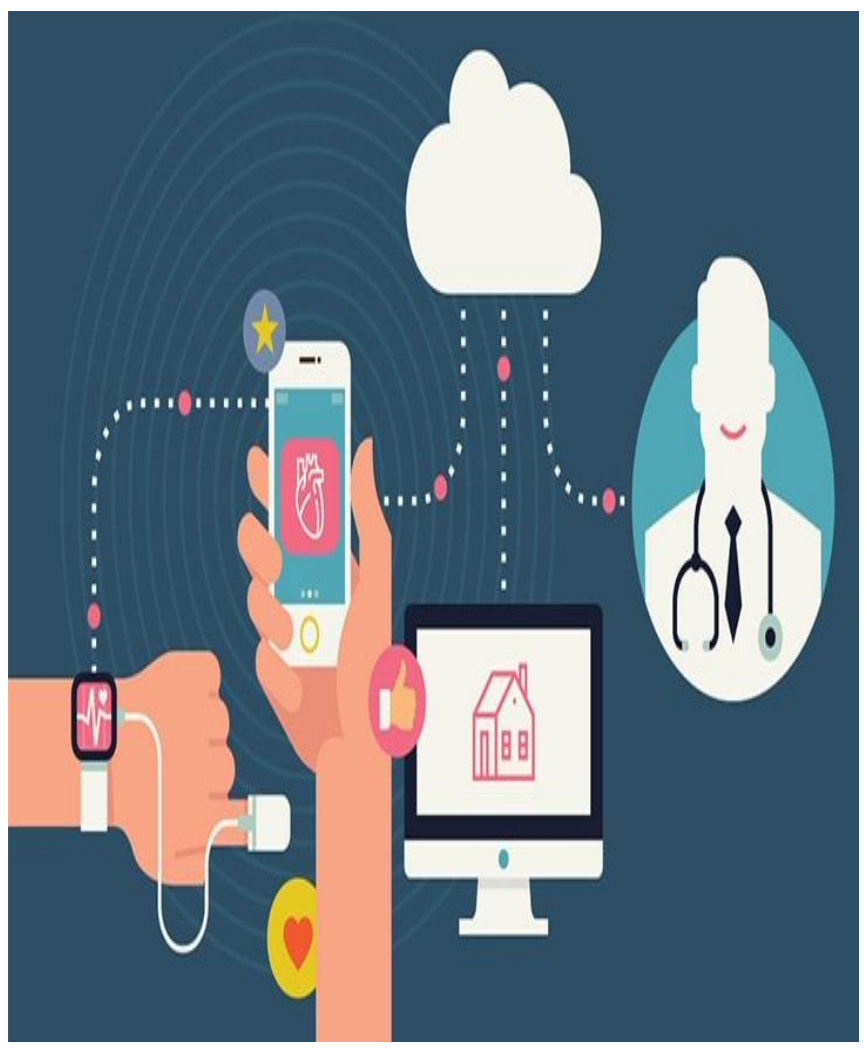

Figure 1: Internet of Things in Present-Day Healthcare Schemes [4] 
In order to aid rapid and timely discovery of the deadly virus, further investigations are required for designs of computerized and active-alert schemes. In this way, both convenient and efficient public-health (PH) actions can be implemented to evade the dangers of the ongoing epidemics and outbreaks. This paper concisely examines IoT technology's potential in tackling the COVID-19 virus by revolutionizing healthcare-systems from traditional to a more tailored system over which a virus can be detected, treated, and supervised with ease.

The rest of this paper is ordered as follows. Section 2 discusses the COVID-19 virus and IoT technology's significance to tackle the virus, while Section 3 concludes the work.

\section{INTERNET OF THINGS TO UNRAVEL AND TACKLE COVID-19 PANDEMIC}

Since the beginning of 2020, the globe has battled with the COVID-19 epidemic whilst almost every strides to ascertain quick cure for the virus has been defective. Naturally, the virus conception period spans between 1-14 days, whilst the recovery-time spans from 6 to 41 days. The virus can be transmitted from a negative-person with no visible symptoms. IoT technology's integration can be regarded as a secure and dependable direction toward managing the deadly COVID-19 virus.

Lately, the technology of IoT has gained meaningful use and acceptance in healthcare application. Due to the present high exigency of COVID-19, the necessities for monitoring of COVID-19 patients remotely have become indispensable [10]. Figure 2 shows some potential advantages of IoT in COVID-19 virus management.

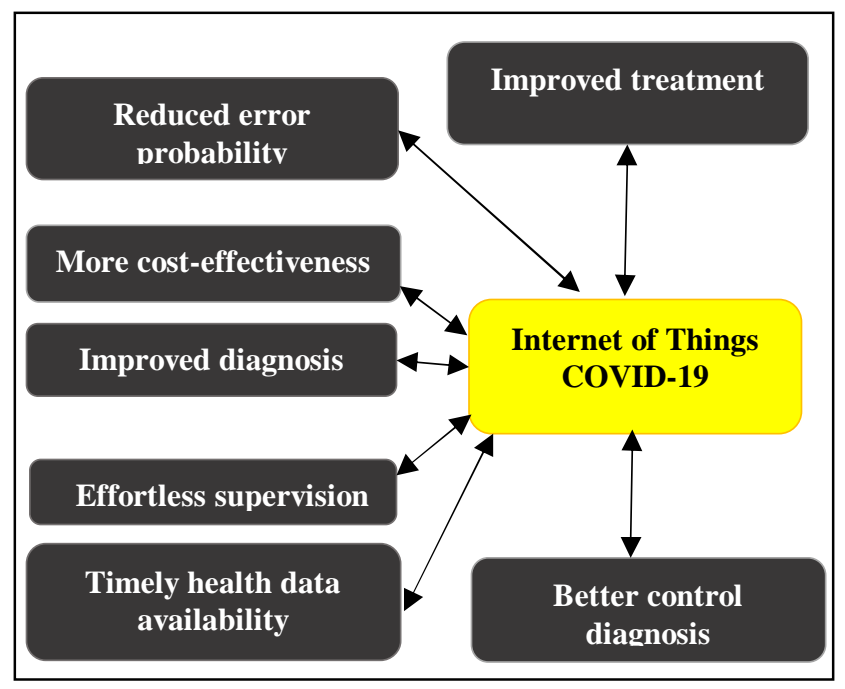

Figure 2: Advantages of Internet of Things Applications in COVID-19 Virus Management
There are critical necessities for earlier identification of COVID-19 because of the contagiousness nature of the virus. This will aid more efficient treatment and control of the widespread of the contagious disease. With IoT sensor-resources and infrastructure, the virus identification can be expedited through tracking the positive patient's data. Moreover, IoT-enabled tracking systems can aid remote monitoring and management of a quarantined or isolated patient. IoT-enabled illness monitoring mechanisms can primarily offer instantaneous data-availability and allow remote medical assistance [11].

By using the information tracked from the patient with devices such as mobile-sensors, the severity of the virus can be identified and tackled efficiently. IoT can also assist a virologist in examining more information on the virus and identify people who have been in close contact with a positivepatient. Explicitly, it can guarantee a patient's passivity during isolation and treatment. Presently, the routine analysis of COVID-19 positive victims by doctors and nurses are performed manually. Meanwhile, the internet of things in health care greatly enhances general healthcare practices with contemporary mobile-sensors, cloud computing and wireless technologies.

Through IoT, positive person's temperature readings can be gained and sent to the internet for retrieval and analysis autonomously. Thereby, a doctor can gather timely data for further statistics and curtail the probabilities of additional spread of the disease by avoiding human contact. Obviously, the cutting-edge technology could reduce overheads of current tracking systems such as absolute ineffectiveness in tracking many patients and regions of interest. IoT scalability appears viable for inspecting victims that might call for isolation in a health care facility [12]. The infrastructure of IoT can significantly facilitate medical procedures to handle outbreaks of already existing viruses. The IoT-system must however enable robust tracking, connectivity, and processing and data availability. Meanwhile, social isolation necessities should be discarded in order to bypass chances of reinfections during recovery from the virus.

Primarily, IoT applications in managing COVID-19 virus are significant for both health workers and COVID-19 victims. The joint role of IoT and related emerging technologies can aid rapid identification of the virus and execution of preventive measures. Therefore, to aid rapid detection and treatment of the lethal virus, more studies are mandatory for designs of computerized and active-alert systems. This will enable more efficient public-health activities and bypass the hazard of the ongoing virus outbreaks.

\section{CONCLUSION}

The COVID-19 epidemic has become a focus of worldwide concern. The symptoms of COVID-19 illness include cough, fatigue, and fever, which are indispensable to identify for prompt control and treatment. 
G. Pius Agbulu et al., International Journal of Emerging Trends in Engineering Research, 9(2), February 2021, 148 - 150

The internet of things (IoT) revolution is presently reforming contemporary healthcare schemes by integrating high-tech, commercial, and societal views. Obviously the internet of things can be a practical way to forecast, monitor, and avert the emerging COVID-19 virus. The paper briefly examined IoT technology's potential as a logical and long-term way to confront COVID-19 by revolutionizing healthcare systems from conventional to more advanced healthcare schemes. The study has clearly presented the effectiveness and prospects of IoT to expedite early detection, treatment, and effortless supervision of the deadly COVID-19 virus.

\section{REFERENCES}

1. S.M.-R. Islam,D. Kwak, M.D.-H. Kabir, M. Hossain and K.-S. The internet of things for health care: a comprehensive survey, IEEE Access vol. 3,pp. 678-708,2015

2. WHO . Coronavirus disease (COVID-19). https://bit.ly/2ZU5x08. Accessed January 6. 16, 2021.

3. J.R.-B, Lovelace. Scientists say the coronavirus is at least as deadly as the 1918 flu pandemic.2020 https://www.cnbc.com/berkeley-lovelace-jr/. Accessed January 1, 2021.

4. P. Nasrullah .Internet of things in healthcare: applications, benefits, and challenges, Jan 2021. Available online: https://www.peerbits.com/blog/internet-of-things-health care-applications-benefits-and-challenges.html.

5. J. Qi, P. Yang, G. Min, O. Amft, F. Dong and L. Xu .Advanced internet of things for personalised healthcare systems: a survey. Pervasive Mob. Comput., vol 41, pp. 132-149,2020.

6. F. Hu, D. Xie and S. Shen. On the application of the internet of things in the field of medical and health care. in Proc. 2013 IEEE International Conference on Green Computing and Communications an IEEE Internet of Things and IEEE Cyber, Physical and Social Computing.,2013, pp 2053-2058

7. J.Wan , M.A. Al-awlaqi, Li M, et al. Wearable IoT enabled real-time health monitoring system. EURASIP J. Wirel. Commun. Netw. vol . 2018,no 1 pp. 298.2020.

8. E. Christaki. New technologies in predicting, preventing and controlling emerging infectious diseases. Virulence, vol. 6 no.6, pp. 558-65,2015.

9. S. Nazir ,Y. Ali, N. Ullah and I. García-Magariño Internet of Things for healthcare using effects of mobile computing: a systematic literature review. Wirel Commun. Mob. Comput. vol.2019, 2019.

10. N. Chen ,M. Zhou M , X. Dong et al. Epidemiological and clinical characteristics of 99 cases of 2019 novel coronavirus pneumonia in Wuhan, China: a descriptive study. Lancet 2020.

11. IoT in healthcare market. 2020 https://www.marketsandmarkets.com/Market-Reports/io t-healthcaremarket-160082804.html.

Accessed December 30, 2020.

12. K. Ashton et al. That internet of thing. RFID J. vol. 22,no. 7, pp. 97-114,2009. 